\title{
LIMIT OF LATTICES IN A LIE GROUP
}

\author{
BY \\ S. P. WANG( $\left.{ }^{1}\right)$
}

1. Introduction. Let $G$ be a connected Lie group, $\Gamma$ a discrete subgroup and $G / \Gamma$ be the space of left cosets. Let $\mu$ be a right Haar measure of $G$. $\mu$ induces a measure $\bar{\mu}$ over $G / \Gamma$. If $\bar{\mu}(G / \Gamma)$ is finite, $\Gamma$ is called a lattice. If $G / \Gamma$ is compact, $\Gamma$, certainly being a lattice, is called a $c$-lattice. We use $A(G)$ to denote the group of all continuous automorphisms of $G$ with compact open topology and $\mathscr{S}(G)\left(\mathscr{S}_{c}(G)\right)$ to denote the set of all lattices (c-lattices) of $G$. We give $\mathscr{S}(G)$ a topology induced from limit of lattices [2]. We know that $A(G)$ operates continuously on $\mathscr{S}(G)$. In [2], Chabauty conjectured that for a lattice $\Gamma_{0}$ of $G, A(G) \Gamma_{0}$ with topology induced from $\mathscr{S}(G)$ is homeomorphic to $A(G) / N\left(\Gamma_{0}\right)$ where $N\left(\Gamma_{0}\right)$ is the subgroup $\left\{\alpha: \alpha \in A(G), \alpha\left(\Gamma_{0}\right)=\Gamma_{0}\right\}$. The purpose of this paper is to study $\mathscr{S}(G)$ and Chabauty's conjecture.

1.1. Definition. A set $\left\{H_{\lambda}\right\}$ of subgroups of $G$ is called uniformly discrete if $H_{\lambda} \cap V=\{e\}$ for a certain neighborhood $V$ of the identity $e$ and all $\lambda$.

1.2. Definition. A sequence $\left\{H_{n}\right\}$ of subgroups of $G$ converges to a subgroup $H$, with notation $\lim H_{n}=H$, if given any compact subset $K$ and neighborhood $V$ of $e$, $H \cap K \subset V H_{n}$ and $H_{n} \cap K \subset V H$ holds for large $n$.

As a consequence of the definitions, we have the following

1.3. LEMMA. Let $\left\{H_{n}\right\}$ be a sequence of subgroups of $G$ converging to a discrete subgroup $H$; then $\left\{H_{n}\right\}_{n \geqq n_{0}}, n_{0}$ a certain integer, is uniformly discrete.

Proof. Let $V$ be a small compact neighborhood of $e$ such that $V^{-1} V \cap H=\{e\}$. Since $e \notin V(H-\{e\})$ and $V(H-\{e\})$ is closed, there exists a neighborhood $W$ of $e$ disjoint with $V(H-\{e\})$. Choose a symmetric compact neighborhood $K$ of $e$ and a symmetric neighborhood $L$ of $e$ such that $K \subset V \cap W$ and $L^{2} \subset K$. As $\lim H_{n}=H$, $H_{n} \cap K \subset L H$ holds for large $n$. Let $n$ be sufficiently large and $x, y \in H_{n} \cap K$, then $x, y \in L$ for $K \cap L(H-\{e\})=\varnothing$. Hence $x^{-1} y \in H_{n} \cap L^{2} \subset H_{n} \cap K$ and $H_{n} \cap K$ is a group. But $K$ is a small neighborhood of $e, H_{n} \cap K=\{e\}$ for large $n$.

1.4. LeMmA. (6) Let $\left\{\Gamma_{n}\right\}$ be a sequence of subgroups of $G$ converging to a c-lattice $\Gamma$ of $G$; then there exists a compact subset $K$ with $G=K \Gamma_{n}$ for large $n$.

2. A base for the topology of $\mathscr{S}(G)$. Let $K$ be a compact subset of $G, V$ be a neighborhood of $e$ and $\Gamma_{0}$ be a lattice of $G$. We define $W\left(K, V: \Gamma_{0}\right)=\{\Gamma: \Gamma \in \mathscr{S}(G)$, $\Gamma \cap K \subset V \Gamma_{0}$ and $\left.\Gamma_{0} \cap K \subset V \Gamma\right\}$. It is easy to see that the family of all $W\left(K, V: \Gamma_{0}\right)$

Received by the editors April 21, 1967.

( ${ }^{1}$ ) Work supported in part by NSF Grant GP 6556. 
with $K$ running through all compact subsets of $G, V$ through all open neighborhoods of $e$ and $\Gamma_{0}$ through $\mathscr{S}(G)$ forms a base for the topology of $\mathscr{S}(G)$ induced from limit of lattices.

\subsection{Proposition. $\mathscr{S}(G)$ is a Hausdorff space.}

Proof. Given any two lattices $\Gamma_{1} \neq \Gamma_{2}$, we may assume that there is an element $\gamma_{1} \in \Gamma_{1}-\Gamma_{2}$. Let $W$ be a relative compact, symmetric open neighborhood of $e$ such that $\bar{W}^{2} \gamma_{1} \cap \Gamma_{2}=\varnothing$. Then $W\left(\left\{\gamma_{1}\right\}, W: \Gamma_{1}\right)$ and $W\left(\left\{\bar{W} \gamma_{1}, e\right\}, W: \Gamma_{2}\right)$ are disjoint.

2.2. Since $G$ is separable, there exists a countable dense subset $\mathscr{M}=$ $\left\{x_{1}, \ldots, x_{n}, \ldots\right\}$ of $G$. Let $\mathscr{F}(\mathscr{M})$ be the set of all finite subsets of $\mathscr{M}$. Let $K$ be a compact subset, $V$ a neighborhood of $e$ and $A \in \mathscr{F}(\mathscr{M})$, we define $V(A, K, V)$ $=\{\Gamma: \Gamma \in \mathscr{S}(G), \Gamma \cap K \subset V A$ and $A \subset V \Gamma\}$. It is clear that $V(A, K, V) \subset W\left(K, V^{2} ; \Gamma\right)$ for all $\Gamma \in V(A, K, V)$. Now let $\left\{V_{n}\right\}$ be a sequence of relative compact, symmetric open neighborhoods of $e$ such that $V_{n} \supset \bar{V}_{n+1}^{2}$ and $\bigcap_{n=1}^{\infty} V_{n}=\{e\}$, and $\left\{K_{n}\right\}$ be a sequence of compact subsets of $G$ with $e \in K_{n} \subset K_{n+1}$ and $\bigcup_{n=1}^{\infty} K_{n}^{0}=G\left({ }^{2}\right)$.

Proposition. Let $W_{A, n}=W\left(\bar{V}_{n} K_{n}, V_{n+1} ; \Gamma_{A, n}\right)$ if $V\left(A, \bar{V}_{n} K_{n}, V_{n+2}\right)$ is not empty and $\Gamma_{A, n}$ a fixed element in it, =empty otherwise. Then $\left\{W_{A, n}: A \in \mathscr{F}(\mathscr{M})\right.$, $n=1,2, \ldots\}$ is a countable base for the topology of $\mathscr{S}(G)$.

Proof. Let $\Gamma_{0}$ be any lattice of $G$ and $\mathscr{V}$ be any neighborhood of $\Gamma_{0}$ in $\mathscr{S}(G)$. Then $\Gamma_{0} \in W\left(\bar{V}_{n} K_{n}, V_{n+1} ; \Gamma_{0}\right) \subset W\left(K_{n}, V_{n} ; \Gamma_{0}\right) \subset \mathscr{V}$ for certain $n$. Let $\Gamma_{0} \cap \bar{V}_{n} K_{n}$ $=\left\{\gamma_{1}, \ldots, \gamma_{m}\right\}$. Since $\mathscr{M}$ is dense, there exists $A=\left\{x_{i_{1}}, \ldots, x_{i_{m}}\right\}$ such that $\gamma_{j} \in V_{n+2} x_{i,}$, $1 \leqq j \leqq m$. Then $\Gamma_{0} \in V\left(A, \bar{V}_{n} K_{n}, V_{n+2}\right) \subset W\left(\bar{V}_{n} K_{n}, V_{n+1}: \Gamma_{A, n}\right)=W_{A, n}$ and one verifies readily $W_{A, n} \subset \mathscr{V}$.

2.3. By Proposition 2.2, $\mathscr{S}(G)$ is separable and by Proposition $2.1, \mathscr{S}(G)$ is Hausdorff. It is easy to show that $W\left(K, V_{n+1} ; \Gamma\right)^{-} \subset W\left(K, V_{n} ; \Gamma\right)$ which implies that $\mathscr{S}(G)$ is regular. Hence by Urysohn's metrization theorem, we get the following

THEOREM. $\mathscr{S}(G)$ is separable metric.

REMARK. It is still unknown whether $\mathscr{S}(G)$ is locally compact or not. We shall see later that this problem can always be reduced to the case having $G$ semisimple without compact factor.

3. A homeomorphism. Let $G$ be a connected Lie group, $\tilde{G}$ be a covering group of $G$ and $\tilde{G} \stackrel{p}{\rightarrow} G$ be the covering map. We define $p^{*}: \mathscr{S}(G) \rightarrow \mathscr{S}(\tilde{G})$ by $p^{*}(\Gamma)$ $=p^{-1}(\Gamma), \Gamma \in \mathscr{S}(G)$.

3.1. Proposition. $p^{*}$ is a homeomorphism onto a closed subset of $\mathscr{S}(\tilde{G})$.

Proof. It is clear that $p^{*}$ is $1-1$ and $p^{*}(\mathscr{S}(G))=\{\tilde{\Gamma}: \tilde{\Gamma} \in \mathscr{S}(\tilde{G})$ and $\tilde{\Gamma} \supset \operatorname{ker}(p)\}$ is closed.

(2) $X^{0}$ denotes the set of all interior points of $X$. 
Let $\left\{\Gamma_{n}\right\}$ be a sequence of lattices of $G$ converging to a lattice $\Gamma$. We want to verify $\lim p^{-1}\left(\Gamma_{n}\right)=p^{-1}(\Gamma)$. Let $\tilde{K}$ be a compact subset of $\tilde{G}$ and $\tilde{V}$ a neighborhood of $e$ in $\tilde{G}$. Then $\Gamma_{n} \cap p(\tilde{K}) \subset p(\tilde{V}) \Gamma$ and $\Gamma \cap p(\tilde{K}) \subset p(\tilde{V}) \Gamma_{n}$ holds for large $n$. Apply $p^{-1}$ to get the desired inclusion relations. Hence $p^{*}$ is continuous. Similarly one shows that $p^{*-1}$ is continuous.

3.2. Let $G, \tilde{G}, p$ have the same meaning as in $3.1, \Gamma \in \mathscr{S}(G)$ and $\tilde{\Gamma}=p^{-1}(\Gamma)$.

Proposition. If $A(\tilde{G}) \tilde{\Gamma} \cong A(\tilde{G}) / N(\tilde{\Gamma})$, then $A(G) \Gamma \cong A(G) / N(\Gamma)$.

Proof. We identify $\mathscr{S}(G)$ in $\mathscr{S}(\widetilde{G})$ through $p^{*}$ and identify $A(G) \subset A(\widetilde{G})$ by $A(G)$ $=\{\alpha: \alpha \in A(\tilde{G}), \alpha(\operatorname{ker}(p))=\operatorname{ker}(p)\}$. In order to verify this proposition, it suffices to verify that $A(G) \Gamma=A(G) \tilde{\Gamma}$ is locally compact. $\operatorname{ker}(p)$ is finitely generated abelian, say, generated by $\left\{z_{1}, \ldots, z_{m}\right\}$. Let $W$ be a relative compact, symmetric neighborhood of $e$ in $A(\widetilde{G})$ such that for any $w \in W, w z_{i} \in U z_{i}, 1 \leqq i \leqq m$ and $U$ a neighborhood of $e$ in $\tilde{G}$ with $\bar{U} \cap \tilde{\Gamma}=\{e\}$. Claim that $A(G) \tilde{\Gamma} \cap W \tilde{\Gamma}$ is relative compact in $A(G) \tilde{\Gamma}$. Let $\left\{\alpha_{n}\right\}$ be a sequence of elements in $A(G)$ having $\alpha_{n}(\tilde{\Gamma}) \in W \tilde{\Gamma}$ for all $n$. Since $W \tilde{\Gamma}$ is relative compact and $A(\tilde{G}) \tilde{\Gamma} \cong A(\tilde{G}) / N(\tilde{\Gamma})$, there exists $\alpha \in \bar{W}, \beta_{n} \in N(\tilde{\Gamma})$ such that $\left\{\alpha_{n} \beta_{n}\right\}$ (more precisely a subsequence of this) converges to $\alpha$. Then consider $\alpha^{-1}=\lim \beta_{n}^{-1} \alpha_{n}^{-1}, \beta_{n}^{-1} \alpha_{n}^{-1}\left(z_{i}\right) \in \tilde{\Gamma}$ for all $n$. But $\alpha^{-1}\left(z_{i}\right) \in \bar{U} z_{i}$ and $\bar{U} \cap \tilde{\Gamma}$ $=\{e\}$, this implies $\beta_{n}(\operatorname{ker}(p))=\operatorname{ker}(p)$ for large $n$. Hence $\alpha \in A(G)$, and $A(G) \Gamma$ is locally compact at $\Gamma$. By action of $A(G), A(G) \Gamma$ is locally compact. Thus $A(G) \Gamma$ $\cong A(G) / N(\Gamma)$ follows easily.

REMARK. By Proposition 3.2, in order to study Chabauty's conjecture, one can consider only simply connected Lie groups.

4. Representations and limit of lattices. Let $F$ be a free monoid on $m$ generators $\gamma_{1}, \ldots, \gamma_{m}$ and $\left\{w_{1}(\gamma), \ldots, w_{i}(\gamma), \ldots\right\}$ be a set of words in $F$. If $w(\gamma)=\gamma_{j_{1}} \cdots \gamma_{j_{s}}$, we define $W: G^{m} \rightarrow G$ by $w(x)=x_{j_{1}} \cdots x_{j_{s}}$, where $x=\left(x_{1}, \ldots, x_{m}\right) \in G^{m}$.

4.1. LEMMA. If $G=G L(n, R)$, then there exists a positive integer $n_{0}$ such that $W_{i}(x)=e$ for $1 \leqq i \leqq n_{0}$ implies $W_{f}(x)=e$ for all $j\left({ }^{3}\right)$.

Proof. Let $M_{n}(R)$ be the set of all real $n-n$ matrices. We define $\bar{W}_{i}: M_{n}(R)^{m}$ $\rightarrow M_{n}(R)$ by $\bar{W}_{i}(A)=A_{j_{1}} \cdots A_{j_{s}}-E$ where $A=\left(A_{1}, \ldots, A_{m}\right), w_{i}(\gamma)=\gamma_{j_{1}} \cdots \gamma_{j_{s}}$ and $E$ is the identity matrix. It is clear that $W_{i}(X)=E$ iff $\bar{W}_{i}(X)=0$ and $X \in G^{m}$. Let $p_{k l}: M_{n}(R) \rightarrow R E_{k l}$ be the projection which assigns to any matrix its $(k, l)$ entry, and $W_{k l}^{i}=p_{k l} \circ \bar{W}_{i}, 1 \leqq k, l \leqq n$ and $i=1,2, \ldots$ Then $W_{k l}^{i} \in R\left[Y_{1}, \ldots, Y_{n^{2} m}\right]$, the ring of real polynomials on $n^{2} m$ variables. Since $R\left[Y_{1}, \ldots Y_{n^{2}}\right]$ is Noetherian, there exists a positive integer $n_{0}$ such that the ideal generated by $W_{k, l}^{i}, i \leqq n_{0}$ contains all $W_{s, t}^{j}$, and the Lemma follows immediately.

4.2. Let $\Gamma$ be a discrete subgroup of $G$ and $\mathscr{R}(\Gamma, G)$ be the space of all representations, i.e., homomorphisms of $\Gamma$ into $G$ with compact open topology.

$\left({ }^{3}\right)$ This was pointed out to the author by H. C. Wang. 
THEOREM. Let $\left\{\Gamma_{n}\right\}$ be a sequence of subgroups of $G$ converging to a discrete subgroup $\Gamma$ of $G$ and $T$ is a finitely generated subgroup of $G$ contained in $\Gamma$; then there are $r_{n} \in \mathscr{R}(T, G)$ such that

(i) $r_{n}(T) \subset \Gamma_{n}$ and

(ii) $\lim r_{n}=1_{T}$ where $1_{T}$ is the inclusion map of $T$ into $G$.

Proof. Let $\tilde{G} \stackrel{p}{\rightarrow} G$ be the universal covering map and $\tilde{G} \stackrel{q}{\rightarrow} G L(n, R)$ be a continuous homomorphism which is a local isomorphism. We set $\tilde{\Gamma}=p^{-1}(\Gamma)$, $\tilde{T}=p^{-1}(T)$, and $\tilde{\Gamma}_{n}=p^{-1}\left(\Gamma_{n}\right)$ for all $n$. By Lemma $1.3, \Gamma_{n}$ is discrete for large $n$, and by same argument used in $3.1, \lim \tilde{\Gamma}_{n}=\tilde{\Gamma}$. Since $T$ and $\operatorname{ker}(p)$ are finitely generated, $\tilde{T}$ is finitely generated. Hence there exists a finite generating subset $\left\{\tilde{\gamma}_{1}, \ldots, \tilde{\gamma}_{m}\right\}$ of $\tilde{T}$ with a set of fundamental relations $\left\{w_{1}(\tilde{\gamma}), \ldots, w_{i}(\tilde{\gamma}), \ldots\right\}$. By the preceding Lemma, there exists a positive integer $n_{0}$ such that $W_{i}(x)=e, 1 \leqq i \leqq n_{0}$ and $x \in \tilde{G}^{m}$ implies $q W_{j}(x)=e$ for all $j$. Let $\mathscr{R}=\bigcap_{i=1}^{n_{0}} W_{i}^{-1}(e)$; then $\mathscr{R}$ is a real analytic subset of $\tilde{G}^{m}$, hence is locally connected. Let $\mathscr{V}$ be a connected neighborhood of $\tilde{\gamma}=\left(\tilde{\gamma}_{1}, \ldots\right.$ $\left.\tilde{\gamma}_{m}\right)$ in $\mathscr{R}$. Since $\operatorname{ker}(q)$ is discrete, we must have that $W_{i}(x)=e$ for all $x \in \mathscr{V}$. Since $\lim \tilde{\Gamma}_{n}=\tilde{\Gamma}$, there are $\gamma_{i}^{(n)} \in \tilde{\Gamma}_{n}, 1 \leqq i \leqq m$ such that $\lim _{n} \gamma_{i}^{(n)}=\tilde{\gamma}_{i}, 1 \leqq i \leqq m$. From Lemma 1.3, we know that $\left\{\tilde{\Gamma}_{n}\right\}_{n>k}$, for certain positive integer $k$, is uniformly discrete. Since $W_{i}\left(\tilde{\gamma}^{(n)}\right) \in \tilde{\Gamma}_{n}, \lim _{n} W_{i}\left(\tilde{\gamma}^{(n)}\right)=W_{i}(\tilde{\gamma})=e$, it follows that $\tilde{\gamma}^{(n)}=\left(\gamma_{1}^{(n)}, \ldots\right.$, $\left.\gamma_{m}^{(n)}\right) \in \mathscr{V}$. Hence there are representations $\tilde{r}_{n}: \tilde{T} \rightarrow \tilde{G}$ such that $\tilde{r}_{n}(\tilde{\gamma})=\tilde{\gamma}^{(n)}$. It is clear that (i) and (ii) are satisfied for $\left\{\tilde{\Gamma}_{n}\right\}, \tilde{\Gamma}, \tilde{T}$ and $\left\{\tilde{r}_{n}\right\}$. But $\operatorname{ker}(p) \subset \tilde{T}, \tilde{\Gamma}$ and $\tilde{\Gamma}_{n}$ for all $n$, we must have $\tilde{r}_{n} \mid \operatorname{ker}(p)=1_{\operatorname{ker}(p)}$. Hence $\tilde{r}_{n}$ induces $r_{n}: T \rightarrow G$ which satisfies (i) and (ii).

5. Stability of subgroups. Let $M$ be a compactly generated, closed, normal subgroup of $G$ with $G / M$ semisimple and having no compact factor, and $M \cap \Gamma$ are $c$-lattices of $M$ for all $\Gamma \in \mathscr{S}(G)$.

Proposition. If $\left\{\Gamma_{n}\right\}$ is a sequence of lattices of $G$ converging to a lattice $\Gamma_{0}$, and $\left\{r_{n}\right\}$ is a sequence of representations of $T_{0} \cap M$ such that (i) $r_{n}\left(\Gamma_{0} \cap M\right) \subset \Gamma_{n}$ for all $n$ and (ii) $\lim r_{n}=1_{\Gamma_{0} \cap M}$, then $r_{n}\left(\Gamma_{0} \cap M\right) \subset M$ for large $n$.

Proof. Let $\rho$ be an irreducible representation of $G / M$ over a complex vector space with $\operatorname{ker}(\rho)=$ center of $G / M$. Such a $\rho$ always exists. Let $\pi: G \rightarrow G / M$ be the projection. Since $\pi\left(\Gamma_{0}\right)$ is a subgroup of $G / M$ with property $(S), C \rho\left(\pi\left(\Gamma_{0}\right)\right)$, the vector space generated by $\rho\left(\pi\left(\Gamma_{0}\right)\right)$, contains $\rho(\pi(G))(1)$. As $C \rho\left(\pi\left(\Gamma_{0}\right)\right)$ is of finite dimension, and $\Gamma_{0} \cap M$, being a $c$-lattice of $M$, is finitely generated, there exists a finitely generated subgroup $T$ of $\Gamma_{0}$ containing $\Gamma_{0} \cap M$ and $C \rho(\pi(T))=C \rho(G / M)$. By Proposition 4.2, there are representations $r_{n}^{\prime}$ of $T$ such that (i) $r_{n}^{\prime}(T) \subset \Gamma_{n}$, (ii) $\lim _{n} r_{n}^{\prime}=1_{T}$ and (iii) $r_{n}^{\prime} \mid \Gamma_{0} \cap M=r_{n}$ for large $n$, and (iv) $C \rho\left(\pi r_{n}^{\prime}(T)\right)=C \rho(G / M)$ for large $n$. Assume that $n$ is sufficiently large. Choose a neighborhood $U_{1}$ of $e$ in $G / M$ with $\lim _{m} L_{m}\left(U_{1}\right)=e$ (for definition see $\left[9\right.$, p. 210]) and $U_{1}$ containing no central element $\neq e, U=\pi^{-1}\left(U_{1}\right) . \Gamma_{0} \cap M$, a $c$-lattice, has a finite generating subset $Q=\left\{\beta_{1}, \ldots, \beta_{s}\right\}$. Hence $\beta_{i}^{-1} r_{n}\left(\beta_{i}\right) \in U$ for $1 \leqq i \leqq s, \lim _{m} L_{m}\left(\pi r_{n}(Q)\right) \subset \lim _{m} L_{m}\left(U_{1}\right)=e$. 
But $\pi r_{n}\left(M \cap \Gamma_{0}\right) \subset \pi\left(\Gamma_{n}\right)$ is discrete. By a slight modification of [9, 3.2], $\pi r_{n}\left(M \cap \Gamma_{0}\right)$ being nilpotent and normal in $\pi r_{n}^{\prime}(T)$, is central. Since $Q$ generates $M \cap \Gamma_{0}$ and $\pi r_{n}(Q)=e$, we get $r_{n}\left(M \cap \Gamma_{0}\right) \subset M$ for large $n$.

6. In this section we deal with some properties of Haar measure. Let $H$ be a locally compact and $\sigma$-compact group, $\Gamma$ a lattice of $H, K$ a closed subgroup containing $\Gamma$, and $H \stackrel{\theta}{\rightarrow} H / K$ be the projection.

\subsection{Lemma. If $\theta$ has a local cross section, then $\Gamma$ is a lattice of $K$.}

Proof. Let $V$ be a relative compact, open neighborhood of $e K$ in $G / K$ and $s$ be a local cross section defined over $\bar{V}$. Given a right Haar measure $\mu$ of $H$, we define $\mu_{v}(B)=\mu(s(V) B)$ for any Borel subset $B$ in $K$. It is easy to verify that $\mu_{v}$ is a right Haar measure of $K$. Let $F$ be a fundamental domain in $K$ with respect to $\Gamma$. Then $s(V) F$ is a $\Gamma$-packing in $G$, i.e. $F^{-1} s(v)^{-1} s(v) F \cap \Gamma=\{e\}$. Hence $\mu_{v}(F)=\mu(s(v) F)$ $\leqq \bar{\mu}(G / \Gamma)<\infty$, and $\Gamma$ is a lattice of $K$.

6.2. LEMMA. Let $G$ be a connected Lie group, $H$ a closed normal subgroup and $G \stackrel{\pi}{\rightarrow} G / H$ be the projection. If $\mathscr{S}(G) \neq \varnothing$ and $\pi(\Gamma)$ is discrete for all $\Gamma \in \mathscr{S}(G)$, then there exist Haar measures $\mu_{G}, \mu_{H}, \mu_{G / H}$ such that $\bar{\mu}_{G}(G / \Gamma)=\bar{\mu}_{G / H}(G / \Gamma H) \bar{\mu}_{H}(H / \Gamma \cap H)$ for all $\Gamma \in \mathscr{S}(G)$.

Proof. Since $\mathscr{S}(G) \neq \varnothing$ and $\pi(\Gamma)$ is discrete for $\Gamma \in \mathscr{S}(G)$, we must have that $H$ and $G$ are unimodular. We define $\mu_{G}$ by

$$
\int_{G} f(x) d \mu_{G}(x)=\int_{G / H} \int_{H} f(x h) d \mu_{H}(h) d \mu_{G / H}(x H)
$$

where $\mu_{H}$ and $\mu_{G / H}$ are arbitrary. It is clear that $\mu_{G}$, so defined, is a Haar measure. Given any $\Gamma \in \mathscr{S}(G)$, there are Borel subsets $F_{1}, F_{2}$ such that

(i) $F_{2}$ is a fundamental domain of $\Gamma \cap H$ in $H$,

(ii) $F_{1}^{-1} F_{1} \cap H \Gamma=\{e\}$,

(iii) $F_{1} F_{2} \Gamma=G$.

It is clear that $F_{1} F_{2}$ is a fundamental domain of $\Gamma$ in $G$, and $F_{1} H$ is a fundamental domain of $\Gamma H$ in $G / H$. Hence by formula $(1), \bar{\mu}_{G}(G / \Gamma)=\bar{\mu}_{G / H}(G / \Gamma H) \bar{\mu}_{H}(H / \Gamma \cap H)$ is immediate.

REMARK. The lemma is true for any discrete subgroup $\Gamma$ of $G$ with $\pi(\Gamma)$ discrete.

7. Some continuous maps. Let $G$ be a simply connected Lie group, $R$ the radical of $G$, and $C$ be the maximal compact normal subgroup of a semisimple part of $G$.

7.1. Lemma [4]. Let $\Gamma \in \mathscr{S}(G)$, then $\Gamma \cap C R$ is a c-lattice of $C R$.

7.2. Proposition. Let $q: \mathscr{S}(G) \rightarrow \mathscr{S}(C R)$ be defined by $q(\Gamma)=\Gamma \cap C R, \Gamma \in \mathscr{S}(G)$. Then $q$ is continuous.

Proof. Immediate from Theorem 4.2.

7.3. By 6.2, we know that $\Gamma C R$ is a lattice of $G / C R$ for any $\Gamma \in \mathscr{S}(G)$. We define $\mathscr{S}(G) \stackrel{p}{\rightarrow} \mathscr{S}(G / C R)$ by $p(\Gamma)=\Gamma C R, \Gamma \in \mathscr{S}(G)$. 
Proposition. $p$ is continuous.

Proof. Let $\left\{\Gamma_{n}\right\}$ be a sequence of lattices of $G$ converging to $\Gamma \in \mathscr{S}(G)$. Since $\lim \left(\Gamma_{n} \cap C R\right)=\Gamma \cap C R$ in $\mathscr{S}(C R)$, and $\Gamma \cap C R$ is a $c$-lattice of $C R$, by Lemma 1.4, there exists a compact subset $K_{1}$ of $C R$ with $K_{1}\left(\Gamma_{n} \cap C R\right)=C R$ for all $n$. Let $K$ be any compact subset of $G, V$ a neighborhood of $e$ in $G$. Then $\Gamma_{n} C R \cap K C R$ $=\left(\Gamma_{n} K_{1}^{-1} \cap K\right) C R \subset\left(\Gamma_{n} \cap K K_{1}\right) C R \subset V \Gamma C R$ and $\Gamma C R \cap K C R=\left(\Gamma K_{1}^{-1} \cap K\right) C R$ $\subset\left(\Gamma \cap K K_{1}\right) C R \subset V \Gamma_{n} C R$ holds for large $n$. Hence $p$ is continuous.

7.4. Since $G$ is simply connected, $G$ takes the form of a semidirect product $G=G_{1} C R, G_{1} \cong G / C R$. We define $f: \mathscr{S}(G) \rightarrow \mathscr{S}\left(G_{1}\right) \times \mathscr{S}(C R)$ by $f(\Gamma)=(p(\Gamma)$, $q(\Gamma)), \Gamma \in \mathscr{S}(G)$.

LEMMA. Let $\left\{\Gamma_{\lambda}\right\}$ be a set of lattices of $G$, if $\left\{p\left(\Gamma_{\lambda}\right)\right\}$ and $\left\{q\left(\Gamma_{\lambda}\right)\right\}$ are uniformly discrete in $G_{1}, C R$ respectively, then $\left\{\Gamma_{\lambda}\right\}$ is uniformly discrete in $G$.

Proof. Immediate.

REMARK. Let $\left(\Gamma^{\prime}, \Gamma^{\prime \prime}\right) \in \mathscr{S}\left(G_{1}\right) \times \mathscr{S}(C R) . f^{-1}\left(\Gamma^{\prime}, \Gamma^{\prime \prime}\right)$ is uniformly discrete. By 6.2, $\bar{\mu}(G / \Gamma)$ is constant for each $\Gamma \in f^{-1}\left(\Gamma^{\prime}, \Gamma^{\prime \prime}\right)$. Hence by a theorem of Chabauty [2], $f^{-1}\left(\Gamma^{\prime}, \Gamma^{\prime \prime}\right)$ is compact.

7.5. Proposition. Let $\left\{\Gamma_{n}\right\}$ be a sequence of lattices of $G$ with $\left\{f\left(\Gamma_{n}\right)\right\}$ converging to $\left(\Gamma^{\prime}, \Gamma^{\prime \prime}\right)$. Then there exists a subsequence $\left\{\Gamma_{t(n)}\right\}$ converging to a lattice $\Gamma$ of $G$ with $f(\Gamma)=\left(\Gamma^{\prime}, \Gamma^{\prime \prime}\right)$.

Proof. By Lemma 1.3 and Lemma 7.4, $\left\{\Gamma_{n}\right\}$ is uniformly discrete. Hence by a theorem of Chabauty [2], there is a subsequence $\left\{\Gamma_{i(n)}\right\}$ converging to a discrete subgroup $\Gamma$ of $G$. Since $C R$ is closed, and $\lim _{n}\left(\Gamma_{i(n)} \cap C R\right)=\Gamma^{n}$, we must have $\Gamma^{\prime \prime} \subset \Gamma$. By the same argument as used in $7.3, \lim _{n} p\left(\Gamma_{t(n)}\right)=p(\Gamma)=\Gamma^{\prime}$. By the Remark at 6.2, $\Gamma$ is a lattice.

7.6. Corollary. Let $K$ be any compact subset of $\mathscr{S}\left(G_{1}\right) \times \mathscr{S}(C R)$, then $f^{-1}(K)$ is compact in $\mathscr{S}(G)$.

7.7. Corollary. If $\mathscr{S}\left(G_{1}\right)$ is locally compact, then $\mathscr{S}(G)$ is locally compact.

8. A map $v$ from $\mathscr{S}(G)$ to the set of real numbers. Let $\mu$ be a fixed Haar measure of $G$. Consider the map $v$ defined by $v(\Gamma)=\bar{\mu}(G / \Gamma)$ for $\Gamma \in \mathscr{S}(G)$. In general $v$ is not continuous, for an example see [6]. But the following always holds.

8.1. THEOREM [6]. $v \mid \mathscr{S}_{c}(G)$ is continuous and $\mathscr{S}_{c}(G)$ is locally compact $\left.{ }^{4}\right)$.

8.2. Corollary. Let $G$ be a simply connected nilpotent Lie group. If $G$ has a lattice $\Gamma_{0}$, then $\mathscr{S}(G) \cong A(G) / N\left(\Gamma_{0}\right)$.

(4) In [6], that $v \mid \mathscr{S}_{c}(G)$ is continuous was proved. Since $\mathscr{S}_{c}(G)$ is open in $\mathscr{S}(G)$, by a theorem of Chabauty [2], $\mathscr{S}_{c}(G)$ is locally compact. 
Proof. By a theorem of Mal'cev [7], $\mathscr{S}_{c}(G)=\mathscr{S}(G)$ and $A(G) \Gamma_{0}=\mathscr{S}(\mathscr{G})$. Since $A(G)$ and $\mathscr{S}(G)$ are locally compact and $A(G)$ is $\sigma$-compact, we have then $\mathscr{S}(G)$ $\cong A(G) / N\left(\Gamma_{0}\right)$.

8.3. It is known that there is a continuous representation of $A(G)$ into the positive real numbers such that $v(\alpha(\Gamma))=r(\alpha) v(\Gamma)$ for all $\Gamma \in \mathscr{S}(G)$ and $\alpha \in A(G)$.

Proposition. $v \mid A(G) \Gamma$ is continuous.

Proof. Consider $f: \mathscr{S}(G) \rightarrow \mathscr{S}\left(G_{1}\right) \times \mathscr{S}(C R)$. It is clear that $f(A(G) \Gamma) \subset A\left(G_{1}\right) \Gamma^{\prime}$ $\times \mathscr{S}(C R), f(\Gamma)=\left(\Gamma^{\prime}, \Gamma^{\prime \prime}\right)$. By 6.2 , we know that $v(T)=v(p(T)) v(q(T))$, for any $T \in \mathscr{S}(G)$. Since $f, v|\mathscr{S}(C R), v| A\left(G_{1}\right) \Gamma^{\prime}$ are continuous $\left({ }^{5}\right)$, we have $v \mid A(G) \Gamma$ is continuous.

Remark. By Proposition 3.1, Proposition 8.3 is true for all connected Lie groups.

9. Let $\Gamma$ be a finitely generated lattice of $G$ and $\mathscr{R}(\Gamma, G)$ be the space of all representations of $\Gamma$ into $G$. Given any $\alpha \in A(G), r \in \mathscr{R}(\Gamma, G)$ we define $\alpha(r)=\alpha \cdot r$. It is easy to see that $A(G)$ operates continuously on $\mathscr{R}(\Gamma, G)$.

9.1. LEMMA. If $A(G) 1_{\Gamma}$ is a neighborhood of $1_{\Gamma}$ in $\mathscr{R}(\Gamma, G)$, then $A(G) \Gamma$ is open $\mathscr{S}(G)$.

Proof. Suppose false. Then there is a sequence $\left\{\Gamma_{n}\right\}$ of lattices converging to $\Gamma$ with $\Gamma_{n} \notin A(G) \Gamma$ for all $n$. By Theorem 4.2, there are representations $r_{n}$ of $\Gamma$ such that (i) $r_{n}(\Gamma) \subset \Gamma_{n}$ and (ii) $\lim r_{n}=1_{\Gamma}$. By assumption $r_{n} \in A(G)\left(1_{\Gamma}\right)$ for large $n$. Let $\alpha_{n} \in A(G)$ such that $\alpha_{n}\left(1_{\Gamma}\right)=r_{n}$. Then $\alpha_{n}(\Gamma) \subset \Gamma_{n}$ and $\lim \alpha_{n}(\Gamma)=\Gamma$. By Proposition 8.3, $\bar{\mu}(G / \Gamma)=\lim \bar{\mu}\left(G / \alpha_{n}(\Gamma)\right) \geqq \lim \sup _{n} \bar{\mu}\left(G / \Gamma_{n}\right)$. But in [2], Chabauty showed that $\bar{\mu}(G / \Gamma) \leqq \lim \inf _{n} \bar{\mu}\left(G / \Gamma_{n}\right)$. It follows that $\bar{\mu}\left(G / \alpha_{n}(\Gamma)\right)=\bar{\mu}\left(G / \Gamma_{n}\right)$ for large $n$. Hence $\Gamma_{n}=\alpha_{n}(\Gamma)$ for large $n$ which leads to a contradiction.

9.2. LEMMA. If $A(G)\left(1_{\Gamma}\right)$ is a neighborhood of $1_{\Gamma}$ in $\mathscr{R}(\Gamma, G)$, then $A(G) \Gamma \cong$ $A(G) / N(\Gamma)$.

Proof. $A(G) \Gamma$ is open in $\mathscr{S}(G)$. Since $\mathscr{S}(G)$ is metric, $A(G) \Gamma$ is locally closed in $\mathscr{S}(G)$. By Lemma 1.3, and Proposition 8.3, there is a neighborhood $\mathscr{V}$ of $\Gamma$ in $\mathscr{S}(G)$ such that $\bar{V} \subset A(G) \Gamma, \bar{\mu}(G / T)<n_{0}$ for all $T \in \mathscr{V}$ and certain positive number $n_{0}$ and $\mathscr{V}$ is uniformly discrete. By a theorem of Chabauty [2], $\bar{V}$ is compact. Hence $A(G) \Gamma$ is locally compact and $A(G) \Gamma \cong A(G) / N(\Gamma)$.

9.3. Let $\hat{G}$ be the Lie algebra of $G$ and $G$ operates on $\hat{G}$ by means of the adjoint group, $\left\{\gamma_{1}, \ldots, \gamma_{n}\right\}$ a finite generating subset of $\Gamma$ and $\left\{w_{1}(\gamma), \ldots, w_{1}(\gamma), \ldots\right\}$ be a set of fundamental relations. Then $\mathscr{R}(\Gamma, G)$ can be identified with $\bigcap_{i} W_{i}^{-1}(e) \subset G^{m}$ where $W_{i}$ 's are defined in 4. In [9], A. Weil proved that $L=\bigcap_{1} L_{i}, L_{i}=$ the kernel of the tangent mapping to $W_{i}$ at $\left(\gamma_{1}, \ldots, \gamma_{m}\right)$, is the space $Z^{1}(\Gamma, \hat{G})$ of all cocycles of $\Gamma$ in $\hat{G}$. Define $g: A(G) \rightarrow G^{m}$ by $g(\alpha)=\left(\alpha\left(\gamma_{1}\right), \ldots, \alpha\left(\gamma_{m}\right)\right), \alpha \in A(G)$. Let $M$ be

(5) This follows from the fact that $A\left(G_{1}\right)$ is semisimple and $A\left(G_{1}\right) / A\left(G_{1}\right)_{0}$ is finite. 
the image of tangent mapping of $g$ at $e \in A(G)$. By Proposition 4, in [4], $M$ $=\Phi Z^{1}(G, \hat{G})$ where $\Phi: Z^{1}(G, \hat{G}) \rightarrow Z^{1}(\Gamma, \hat{G})$ is the restriction map.

9.4. ThEOREM. If $H^{1}(G, \hat{G}) \stackrel{\oplus^{*}}{\rightarrow} H^{1}(\Gamma, \hat{G})$ is surjective, then the Chabauty's conjecture for $\Gamma$ is true.

Proof. By the above remark, $M=L$, hence by Lemma 1 in $(9), A(G)\left(1_{\Gamma}\right)$ is a neighborhood of $1_{\Gamma}$ in $\mathscr{R}(\Gamma, G)$. Thus by Lemma $9.2, A(G) \Gamma \cong A(G) / N(\Gamma)$.

Acknowledgments. The author is greatly indebted to Professor H. C. Wang for his instruction and valuable suggestions.

\section{REFERENCES}

1. A. Borel, Density properties for certain subgroups of semi-simple groups without compact factors, Ann. of Math. (2) 72 (1960), 179-188.

2. C. Chabauty, Limite d'ensembles et géométrie des nombres, Bull. Soc. Math. France 78 (1950), 143-151.

3. C. Chevalley, Theory of Lie groups. I, Princeton Univ. Press, Princeton, N. J., 1946.

4. H. Garland and M. Goto, Lattices and the adjoint group of a Lie group, Trans. Amer. Math. Soc. 124_(1966), 450-460.

5. S. Helgason, Differential geometry and symmetric spaces, Academic Press, New York, 1962.

6. A. M. Macbeath and S. Swierczkowski, Lattices in a compactly generated group, Canad. J. Math. 7 (1960), 427-438.

7. A. I. Mal'cev, On a class of homogeneous groups, Amer. Math. Soc. Transl. (1) 9 (1951), 276-307.

8. H. C. Wang, On the deformations of lattice in a Lie group, Amer. J. Math. 85 (1963), 189-212.

9. A. Weil, Remarks on the cohomology of groups, Ann. of Math. 80 (1964), 149-158.

INSTITUTE FOR ADVANCED STUDY,

Princeton, New Jersey 